\title{
Characterisations of the transmuted inverse Weibull distribution
}

\author{
M. Shuaib Khan ${ }^{1} \quad$ Robert King ${ }^{2} \quad$ Irene L. Hudson ${ }^{3}$
}

(Received 15 December 2013; revised 16 May 2014)

\begin{abstract}
We characterise the transmuted inverse Weibull distribution and compare it to many other generalizations of the two-parameter inverse Weibull distribution using the likelihood ratio test. Explicit expressions are derived for the quantile, moment generating function, entropies, mean deviation and order statistics. A bladder cancer application is presented to illustrate the proposed transmuted inverse Weibull distribution.
\end{abstract}

\section{Contents}

\section{Introduction}

C198

http://journal.austms.org.au/ojs/index.php/ANZIAMJ/article/view/7785 gives this article, (c) Austral. Mathematical Soc. 2014. Published July 9, 2014, as part of the Proceedings of the 11th Biennial Engineering Mathematics and Applications Conference. ISSN 1446-8735. (Print two pages per sheet of paper.) Copies of this article must not be made otherwise available on the internet; instead link directly to this URL for this article. 
2 Transmuted distribution and transmuted inverse Weibull C200

3 Moments and quantiles

C203

4 Maximum likelihood estimation

C207

5 Entropy and mean deviation

C209

6 Order statistics

C210

7 Bladder cancer application

C211

8 Conclusion

C214

References

C214

\section{Introduction}

Keller and Kamath [9] introduced the inverse Weibull (IW) distribution for modelling reliability data and also discussed this model by investigating failures of mechanical components subject to degradation. The IW distribution is the most popular model to date for analysing life time data and its distribution function is

$$
\mathrm{F}(x ; \alpha, \theta)=\exp \left[-\left(\frac{\theta}{x}\right)^{\alpha}\right], \quad x>0,
$$

where $\alpha>0$ is the shape parameter and $\theta>0$ is the scale parameter. The density function corresponding to (1) is

$$
f(x ; \alpha, \theta)=\alpha \theta^{\alpha} x^{-(\alpha+1)} \exp \left[-\left(\frac{\theta}{x}\right)^{\alpha}\right], \quad x>0 .
$$

The IW distribution can have both increasing and decreasing failure rates, depending on the shape parameter. Calabria and Pulcini [3] obtained the 
maximum likelihood and least squares estimates of the parameters of the IW distribution. Felipe et al. [4] proposed the generalized IW distribution and discussed several properties of this model. Recently, Aryal et al. [2] proposed the transmuted Weibull distribution and discussed its mathematical properties. More recently, Khan and King [7, 8] developed the transmuted modified Weibull and transmuted generalized IW distributions and studied various structural properties of these models using applications. Using the quadratic rank transmutation map studied by Shaw et al. [15], we develop the three parameter transmuted inverse Weibull (TIW) distribution.

The main aims of this article are to introduce the three parameter TIW distribution and compare its properties with the transmuted inverse Rayleigh (TIR) and transmuted inverse exponential (TIE) distributions. The proposed TIW distribution includes five life time distributions as special cases when its parameters are changed. More specifically, the TIW distribution includes as special cases the TIE, TIR, IW, IR (inverse Rayleigh) and IE (inverse exponential) distributions.

In Section 2 we present the behaviour of the density function, cumulative distribution function, reliability function and hazard function of the TIW distribution. Section 3 provides the mathematical properties of the quantile function, moment estimate and moment generating function. In Sections 4-6, we discuss maximum likelihood estimation and inference on the parameters, entropies, mean deviation and the ordinary moments of the order statistics. In Section 7 we fit the TIW distribution to illustrate its usefulness. Concluding remarks are given in Section 8. 


\section{Transmuted distribution and transmuted inverse Weibull}

A random variable $X$ is said to have transmuted distribution if its cumulative distribution function $(\mathrm{CDF})$ is

$$
F(x)=(1+\lambda) G(x)-\lambda G(x)^{2},
$$

where $G(x)$ is the CDF of the base distribution. In new reliability model, a positive random variable $X$ is defined by the $\operatorname{TIW}(x ; \alpha, \theta, \lambda)$ distribution with parameters $\alpha, \theta>0,|\lambda| \leqslant 1$ and probability density function (PDF)

$$
\begin{aligned}
f(x ; \alpha, \theta, \lambda) & =\alpha \theta^{\alpha} x^{-(\alpha+1)} \exp \left[-\left(\frac{\theta}{x}\right)^{\alpha}\right] W(x), \\
W(x) & =1+\lambda-2 \lambda \exp \left[-\left(\frac{\theta}{x}\right)^{\alpha}\right]
\end{aligned}
$$

where $\alpha$ and $\theta$ denote the shape and scale parameters, respectively, and $\lambda$ is the transmuted parameter. The CDF corresponding to (4) is

$$
\mathrm{F}(x ; \alpha, \theta, \lambda)=\exp \left[-\left(\frac{\theta}{x}\right)^{\alpha}\right]\left\{1+\lambda-\lambda \exp \left[-\left(\frac{\theta}{x}\right)^{\alpha}\right]\right\} .
$$

The reliability function (RF), hazard function (HF) and cumulative hazard function ( $\mathrm{CHF}$ ) corresponding to (4) are, respectively,

$$
\begin{aligned}
& \mathrm{R}(x ; \alpha, \theta, \lambda)=1-\exp \left[-\left(\frac{\theta}{x}\right)^{\alpha}\right]\left\{1+\lambda-\lambda \exp \left[-\left(\frac{\theta}{x}\right)^{\alpha}\right]\right\}, \\
& h(x ; \alpha, \theta, \lambda)=\frac{\alpha \theta^{\alpha} x^{-(\alpha+1)} \exp \left[-\left(\frac{\theta}{x}\right)^{\alpha}\right] W(x)}{1-\exp \left[-\left(\frac{\theta}{x}\right)^{\alpha}\right]\left\{1+\lambda-\lambda \exp \left[-\left(\frac{\theta}{x}\right)^{\alpha}\right]\right\}}, \\
& H(x ; \alpha, \theta, \lambda)=-\log [1-F(x ; \alpha, \theta, \lambda)] .
\end{aligned}
$$

Some possible shapes of the PDF, CDF, RF and HF for selected parameter values are shown in Figures 1 and 2. These figure shows the flexibility of the TIW distribution. The HF of the TIW distribution in Figure 2(b) has increasing and decreasing failure rate patterns. 


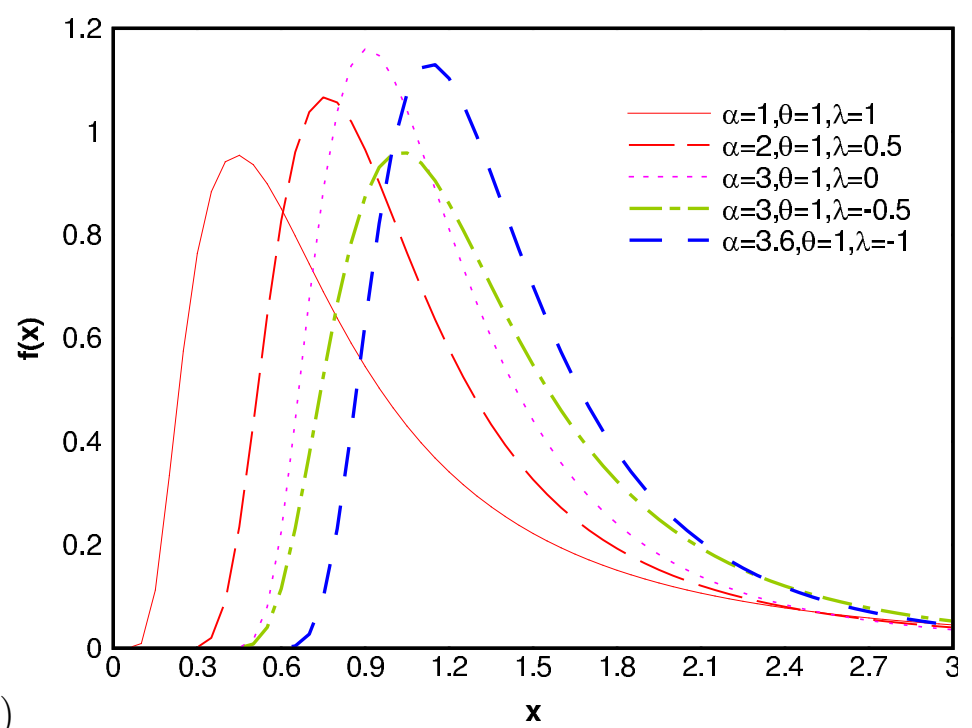

(a)

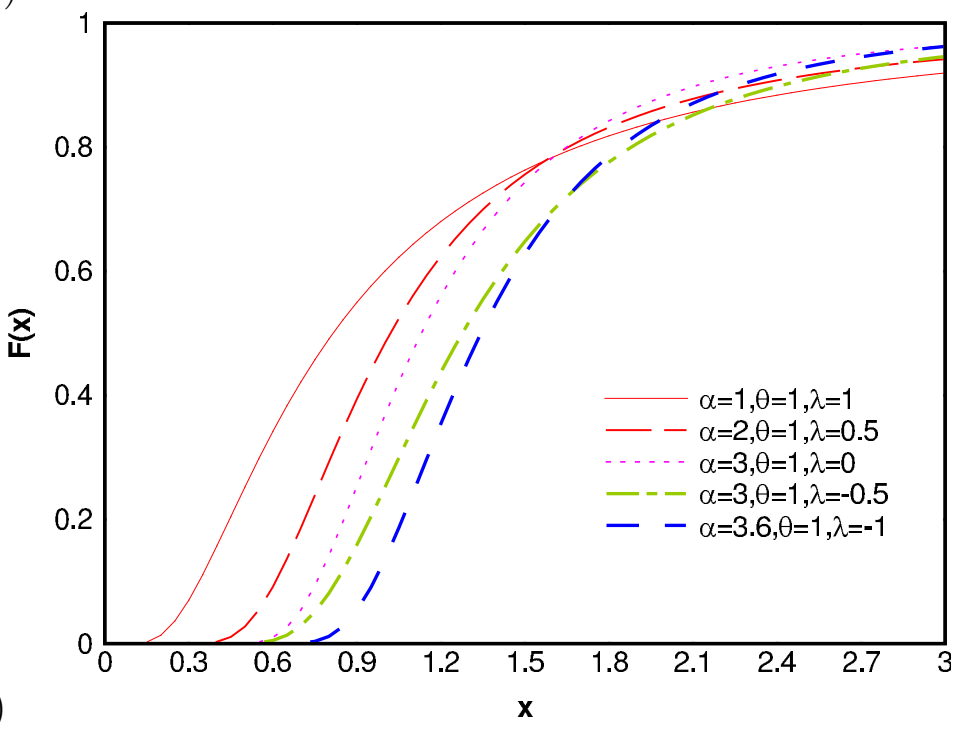

Figure 1: Plots of the TIW (a) PDF; and (b) CDF for some parameter values. 


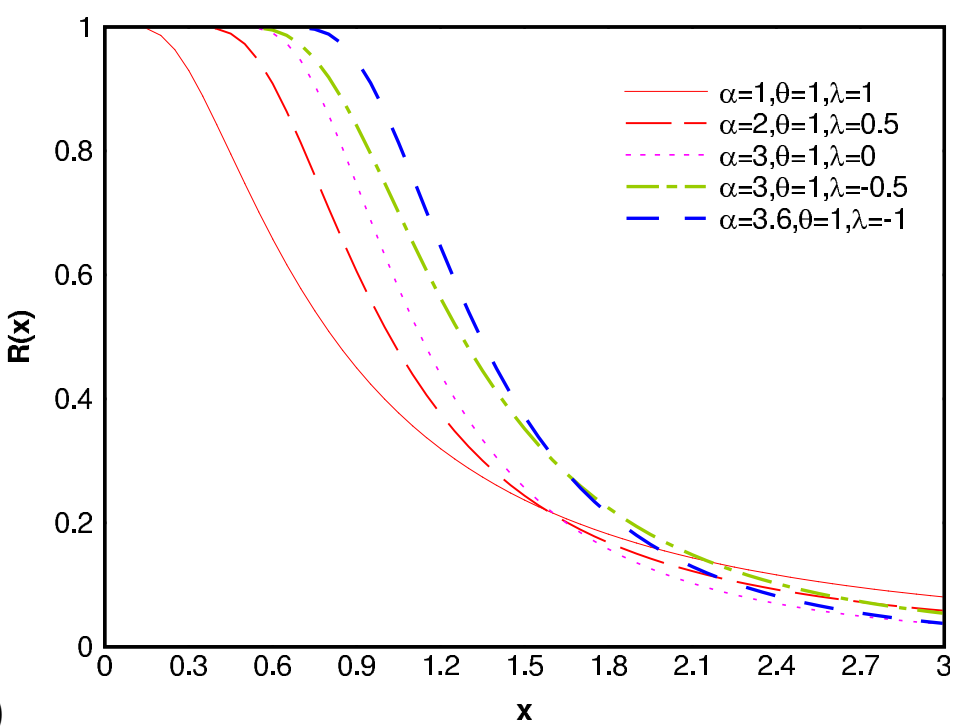

(a)

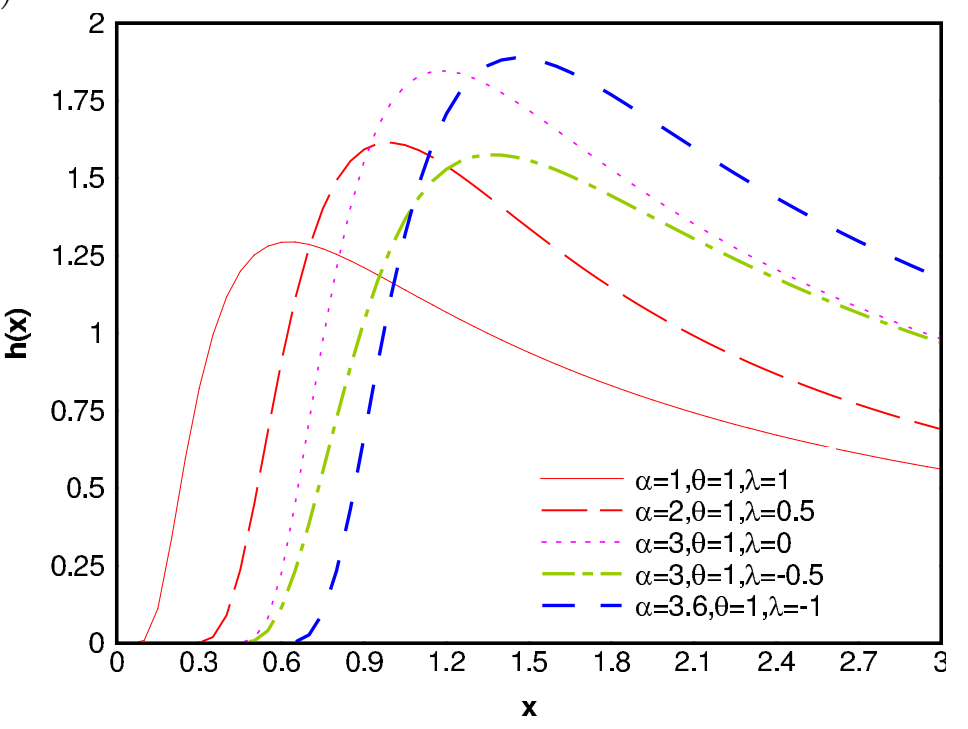

Figure 2: Plots of the TIW (a) RF; and (b) HF for some parameter values. 


\section{Moments and quantiles}

This section presents the kth moment, moment generating function (MGF) and quantile analysis of the $\operatorname{TIW}(x ; \alpha, \theta, \lambda)$ distribution.

Theorem 1. If $\mathrm{X}$ has the $\operatorname{TIW}(x ; \alpha, \theta, \lambda)$ with $|\lambda| \leqslant 1$, then the $\mathrm{k}$ th moment of $\mathrm{x}$ is

$$
\mu_{k}=(1+\lambda) \theta^{k} \Gamma\left(1-\frac{k}{\alpha}\right)-\lambda 2^{k / \alpha} \theta^{k} \Gamma\left(1-\frac{k}{\alpha}\right) .
$$

Proof: From (4) which defines the TIW distribution and from $W(x)$ in (5), we obtain

$$
\mu_{k}=\int_{0}^{\infty} x^{k} \alpha \theta^{\alpha} x^{-(\alpha+1)} \exp \left[-\left(\frac{\theta}{x}\right)^{\alpha}\right] W(x) d x
$$

so that

$$
\begin{aligned}
\mu_{\mathrm{k}}= & (1+\lambda) \int_{0}^{\infty} x^{k} \alpha \theta^{\alpha} x^{-(\alpha+1)} \exp \left[-\left(\frac{\theta}{x}\right)^{\alpha}\right] \mathrm{d} x \\
& -2 \lambda \int_{0}^{\infty} x^{k} \alpha \theta^{\alpha} x^{-(\alpha+1)} \exp \left[-2\left(\frac{\theta}{x}\right)^{\alpha}\right] \mathrm{d} x .
\end{aligned}
$$

The above integral yields the kth moment

$$
\mu_{k}=\theta^{k} \Gamma\left(1-\frac{k}{\alpha}\right)\left[(1+\lambda)-\lambda 2^{k / \alpha}\right] .
$$

Using (10) the mean time to failure (MTTF) and variance $\mathrm{V}(\mathrm{x})$ of the TIW distribution are, respectively,

$$
\begin{aligned}
& \mu_{1}=\theta \Gamma\left(1-\frac{1}{\alpha}\right)\left((1+\lambda)-\lambda 2^{1 / \alpha}\right), \\
& \sigma^{2}=\theta^{2} \Gamma\left(1-\frac{2}{\alpha}\right)\left((1+\lambda)-\lambda 2^{2 / \alpha}\right)-\left(\mu_{1}\right)^{2} .
\end{aligned}
$$


Theorem 2. If $\mathrm{X}$ is a random variable that has the $\operatorname{TIW}(x ; \alpha, \theta, \lambda)$ with $|\lambda| \leqslant 1$, then the moment generating function of $\mathrm{X}$ is

$$
M_{x}(t)=\sum_{m=0}^{\infty} \frac{(t \theta)^{m}}{m !} \Gamma\left(1-\frac{m}{\alpha}\right)\left[(1+\lambda)-\lambda 2^{m / \alpha}\right] .
$$

Proof: By definition

$$
\begin{aligned}
M_{x}(t)= & (1+\lambda) \int_{0}^{\infty} \alpha \theta^{\alpha} x^{-(\alpha+1)} \exp \left[t x-\left(\frac{\theta}{x}\right)^{\alpha}\right] d x \\
& -2 \lambda \int_{0}^{\infty} \alpha \theta^{\alpha} x^{-(\alpha+1)} \exp \left[t x-2\left(\frac{\theta}{x}\right)^{\alpha}\right] d x .
\end{aligned}
$$

Using Taylor series expansions, the above integrals reduce to

$$
\begin{aligned}
M_{x}(t)= & (1+\lambda) \sum_{m=0}^{\infty} \frac{t^{m}}{m !} \alpha \theta^{\alpha} \int_{0}^{\infty} x^{m-\alpha-1} \exp \left[-\left(\frac{\theta}{x}\right)^{\alpha}\right] d x \\
& -2 \lambda \sum_{m=0}^{\infty} \frac{t^{m}}{m !} \alpha \theta^{\alpha} \int_{0}^{\infty} x^{m-\alpha-1} \exp \left[-2\left(\frac{\theta}{x}\right)^{\alpha}\right] d x
\end{aligned}
$$

which yield the following moment generating function

$$
M_{x}(t)=(1+\lambda) \sum_{m=0}^{\infty} \frac{(t \theta)^{m}}{m !} \Gamma\left(1-\frac{m}{\alpha}\right)-\lambda \sum_{m=0}^{\infty} \frac{(t \theta)^{m} 2^{m / \alpha}}{m !} \Gamma\left(1-\frac{m}{\alpha}\right)
$$

Based on Theorem 1, the coefficient of skewness and coefficient of kurtosis of the $\operatorname{TIW}(x ; \alpha, \theta, \lambda)$ are obtained from the well known relations $\beta_{1}=\mu_{3} / \mu_{2}^{\frac{3}{2}}$ and $\beta_{2}=\mu_{4} / \mu_{2}^{2}$, respectively 1 . Figures 3 and 4 illustrate the median, B-life [12], Bowley skewness and Moors kurtosis with different parameter values. 


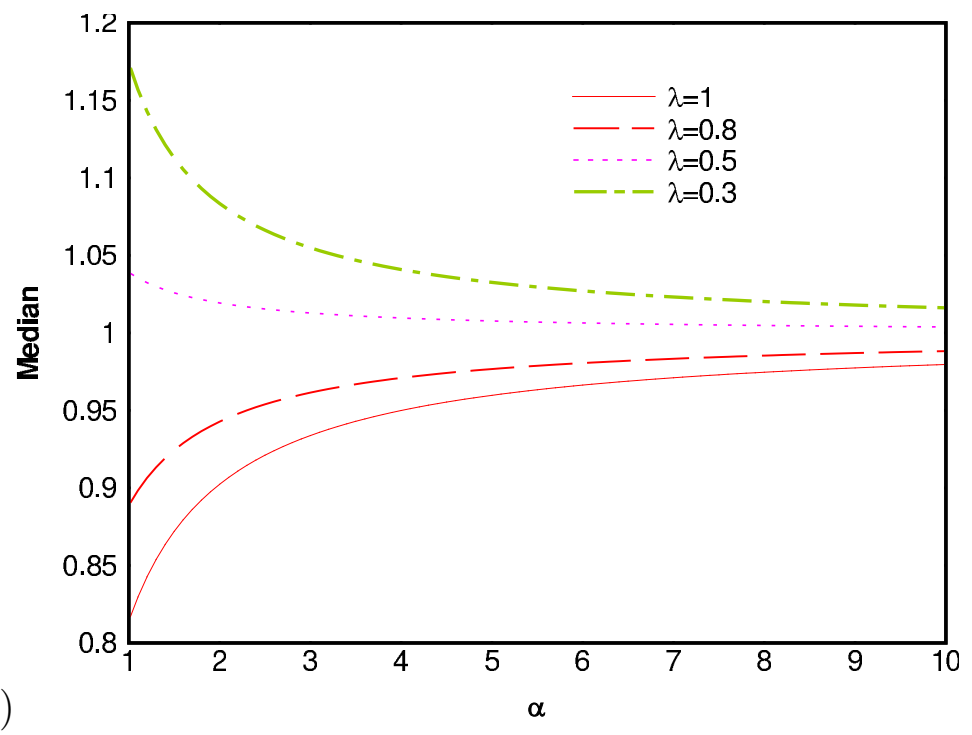

(a)

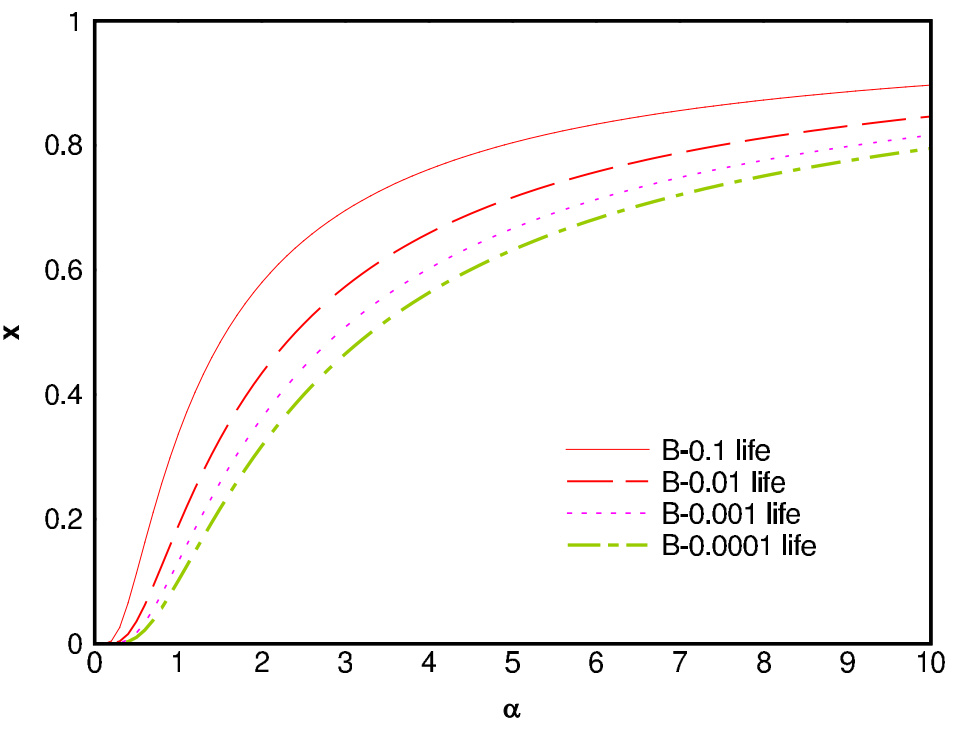

(b)

Figure 3: (a) Median; and (b) B-life of the TIW distribution. 


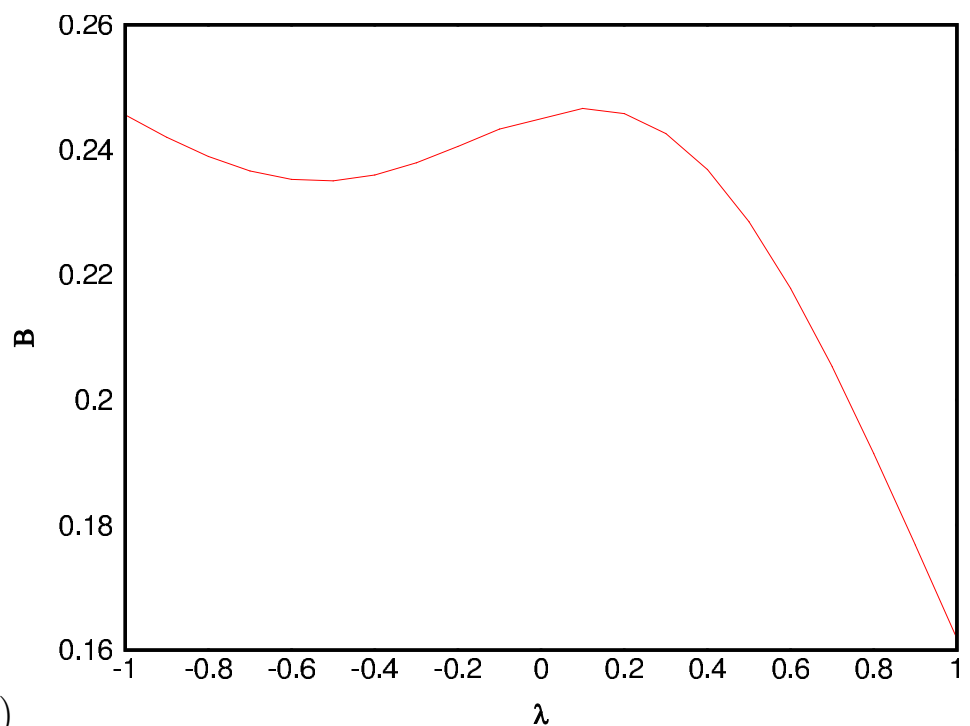

(a)

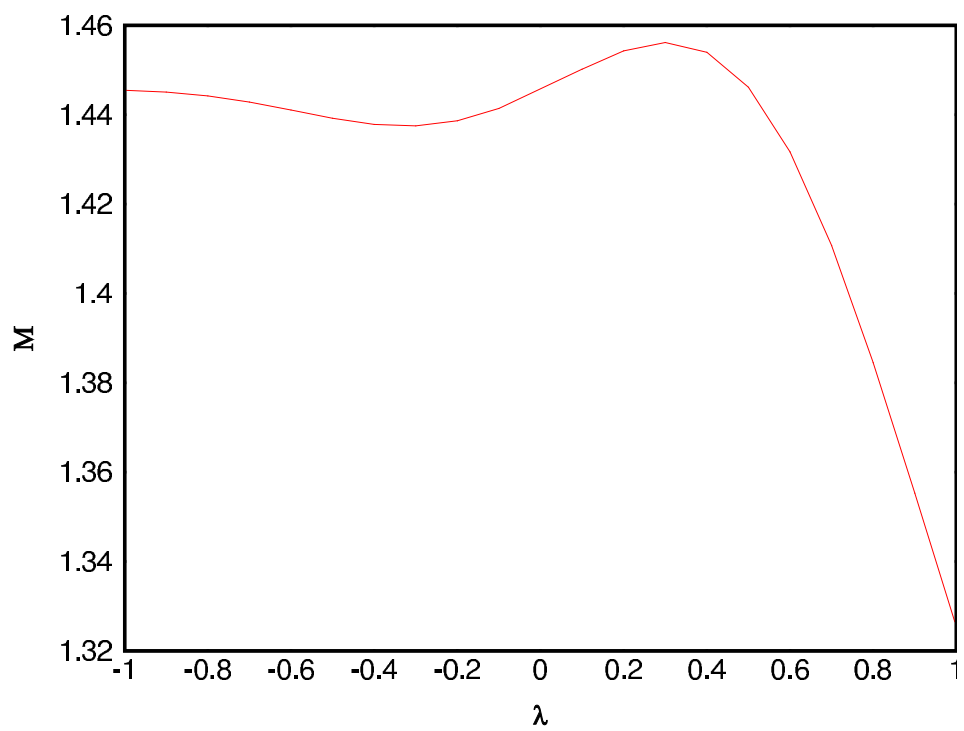

(b)

Figure 4: (a) Bowley skewness; and (b) Moors kurtosis of the TIW distribution. 
The quantile function corresponding to (6) is the real solution of

$$
\mathrm{F}^{-1}(\mathrm{u})=\theta\left[\log \left(\frac{2 \lambda}{(1+\lambda)-\sqrt{(1+\lambda)^{2}-4 \lambda u}}\right)\right]^{-1 / \alpha},
$$

where $u$ has the uniform $\mathrm{u}(0,1)$ distribution. By substituting $u=0.5$ into (14) we obtain the median of the $\operatorname{TIw}(x ; \alpha, \theta, \lambda)$ :

$$
\mathrm{F}^{-1}(0.5)=\theta\left[\log \left(\frac{2 \lambda}{(1+\lambda)-\sqrt{1+\lambda^{2}}}\right)\right]^{-1 / \alpha} .
$$

\section{Maximum likelihood estimation}

The likelihood function of (4) is

$$
\begin{aligned}
\mathrm{L}(\alpha, \theta, \lambda)= & \prod_{i=1}^{n} \alpha \theta^{\alpha} x_{i}^{-(\alpha+1)} \exp \left[-\left(\frac{\theta}{x_{i}}\right)^{\alpha}\right] \\
& \times\left\{1+\lambda-2 \lambda \exp \left[-\left(\frac{\theta}{x_{i}}\right)^{\alpha}\right]\right\} .
\end{aligned}
$$

By taking the logarithm of (16) we obtain the log-likelihood function:

$$
\begin{aligned}
\log L(\alpha, \theta, \lambda)= & n \log \alpha+n \alpha \log \theta-(\alpha+1) \sum_{i=1}^{n} \log \left(x_{i}\right)-\sum_{i=1}^{n}\left(\frac{\theta}{x_{i}}\right)^{\alpha} \\
& +\sum_{i=1}^{n} \log \left\{1+\lambda-2 \lambda \exp \left[-\left(\frac{\theta}{x_{i}}\right)^{\alpha}\right]\right\}
\end{aligned}
$$


Differentiating (17) with respect to $\alpha, \theta$ and $\lambda$ we obtain

$$
\begin{aligned}
\frac{\partial \log L}{\partial \alpha}= & \frac{n}{\alpha}+n \log \theta-\sum_{i=1}^{n} \log \left(x_{i}\right)-\sum_{i=1}^{n}\left(\frac{\theta}{x_{i}}\right)^{\alpha} \log \left(\frac{\theta}{x_{i}}\right) \\
& +2 \lambda \sum_{i=1}^{n} \frac{\exp \left[-\left(\frac{\theta}{x_{i}}\right)^{\alpha}\right]\left(\frac{\theta}{x_{i}}\right)^{\alpha} \log \left(\frac{\theta}{x_{i}}\right)}{1+\lambda-2 \lambda \exp \left[-\left(\frac{\theta}{x_{i}}\right)^{\alpha}\right]} \\
\frac{\partial \log L}{\partial \theta}= & \frac{n \alpha}{\theta}-\left(\frac{\alpha}{\theta}\right) \sum_{i=1}^{n}\left(\frac{\theta}{x_{i}}\right)^{\alpha} \\
& +2 \lambda \sum_{i=1}^{n} \frac{\exp \left[-\left(\frac{\theta}{x_{i}}\right)^{\alpha}\right]\left(\frac{\theta}{x_{i}}\right)^{\alpha}\left(\frac{\alpha}{\theta}\right)}{1+\lambda-2 \lambda \exp \left[-\left(\frac{\theta}{x_{i}}\right)^{\alpha}\right]} \\
\frac{\partial \log L}{\partial \lambda}= & \sum_{i=1}^{n} \frac{1-2 \exp \left[-\left(\frac{\theta}{x_{i}}\right)^{\alpha}\right]}{1+\lambda-2 \lambda \exp \left[-\left(\frac{\theta}{x_{i}}\right)^{\alpha}\right]} .
\end{aligned}
$$

The $3 \times 3$ observed information matrix is

$$
\left[\begin{array}{l}
\hat{\alpha} \\
\hat{\theta} \\
\hat{\lambda}
\end{array}\right] \sim N\left(\left[\begin{array}{l}
\alpha \\
\theta \\
\lambda
\end{array}\right],\left[\begin{array}{lll}
\widehat{\nabla}_{11} & \widehat{\nabla}_{12} & \widehat{\nabla}_{13} \\
\widehat{\nabla}_{21} & \widehat{\nabla}_{22} & \widehat{\nabla}_{23} \\
\widehat{\nabla}_{31} & \widehat{\nabla}_{32} & \widehat{\nabla}_{33}
\end{array}\right]\right)
$$

where

$$
\mathrm{V}^{-1}=-\mathrm{E}\left[\begin{array}{ccc}
\partial^{2} \log \mathrm{L} / \partial \alpha^{2} & \partial^{2} \log \mathrm{L} / \partial \alpha \partial \theta & \partial^{2} \log \mathrm{L} / \partial \alpha \partial \lambda \\
\partial^{2} \log \mathrm{L} / \partial \theta \partial \alpha & \partial^{2} \log \mathrm{L} / \partial \theta^{2} & \partial^{2} \log \mathrm{L} / \partial \theta \partial \lambda \\
\partial^{2} \log \mathrm{L} / \partial \alpha \partial \lambda & \partial^{2} \log \mathrm{L} / \partial \lambda \partial \theta & \partial^{2} \log \mathrm{L} / \partial \lambda^{2}
\end{array}\right]
$$

Solving the inverse matrix of the observed information matrix (21) yields the asymptotic variance and co-variances of the ML estimators $\hat{\alpha}, \hat{\theta}$ and $\hat{\lambda}$. Thus, approximate $100(1-\alpha) \%$ asymptotic confidence intervals for $\alpha, \theta$ and $\lambda$ are

$$
\hat{\alpha} \pm Z_{\frac{\alpha}{2}} \sqrt{\widehat{V}_{11}}, \quad \hat{\theta} \pm Z_{\frac{\alpha}{2}} \sqrt{\widehat{V}_{22}}, \quad \hat{\lambda} \pm Z_{\frac{\alpha}{2}} \sqrt{\widehat{V}_{33}}
$$

where $Z_{\frac{\alpha}{2}}$ is the upper $\alpha$ th percentile of the standard normal distribution. 


\section{$5 \quad$ Entropy and mean deviation}

The Rényi entropy [13], $I_{R}(\rho)$, for $X$ is a measure of variation of uncertainty and is defined as

$$
I_{R}(\rho)=\frac{1}{1-\rho} \log \left[\int_{0}^{\infty} f(x)^{\rho} d x\right],
$$

where $\rho>0$ and $\rho \neq 1$. Suppose $X$ has the $\operatorname{Tiw}(x ; \alpha, \theta, \lambda)$ distribution, then, by substituting (4) and (5) in (22), we obtain

$$
I_{R}(\rho)=\frac{1}{1-\rho} \log \left\{\int_{0}^{\infty} \alpha^{\rho} \theta^{\alpha \rho} x^{-\rho(\alpha+1)} \exp \left[-\rho\left(\frac{\theta}{x}\right)^{\alpha}\right] W(x)^{\rho} d x\right\} .
$$

By solving (23), the TIW Rényi entropy is

$$
\begin{aligned}
\mathrm{I}_{\mathrm{R}}(\rho)= & \frac{\rho}{1-\rho} \log \alpha+\frac{\rho \alpha}{1-\rho} \log \theta+\frac{\rho}{1-\rho} \log (1+\lambda) \\
& +\frac{\rho}{1-\rho} \log \left[\sum_{k=0}^{\infty} C_{\rho: k}\left(\frac{2 \lambda}{1+\lambda}\right)^{k} \frac{(-1)^{k} \Gamma\left(\frac{(\alpha+1)(\rho-1)}{\alpha}+1\right)}{\theta^{(\alpha+1)(\rho-1)+\alpha}(\rho+k)^{\frac{(\alpha+1)(\rho-1)}{\alpha}+1}}\right] .
\end{aligned}
$$

The $\beta$-entropy (or $\alpha$-entropy) was introduced by Havrda and Charvat [6] and is defined as

$$
I_{H}(\beta)=\frac{1}{\beta-1}\left[1-\int_{0}^{\infty} f(x)^{\beta} d x\right],
$$

where $\beta>0$ and $\beta \neq 1$. Suppose $X$ has the $\operatorname{TIW}(x ; \alpha, \theta, \lambda)$ distribution, then, by substituting (4) and (5) in (24), we obtain

$$
\mathrm{I}_{H}(\beta)=\frac{1}{\beta-1}\left\{1-\int_{0}^{\infty} \alpha^{\beta} \theta^{\alpha \beta} x^{-\beta(\alpha+1)} \exp \left[-\beta\left(\frac{\theta}{x}\right)^{\alpha}\right] \mathrm{W}(x)^{\beta} \mathrm{d} x\right\} .
$$

The above integral yields the TIW $\beta$-entropy:

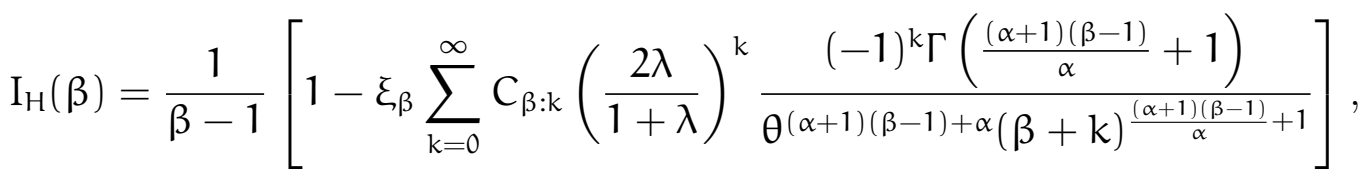


where $\xi_{\beta}=\alpha^{\beta} \theta^{\alpha \beta}(1+\lambda)^{\beta}$.

The degree of scatter in a population is generally measured by the totality of deviations from the mean and median. If $\mathrm{X}$ has the $\operatorname{TIW}(x ; \alpha, \theta, \lambda)$ distribution, then, like Gauss et al. [5], we derive the mean deviation about the mean and about the median from

$$
\delta_{1}=\int_{0}^{\infty}|x-\mu| f(x) d x \quad \text { and } \quad \delta_{2}=\int_{0}^{\infty}|x-M| f(x) d x .
$$

The mean $\mu$ is given in equation (11) and the median $M$ is obtained from equation (15). These measures are calculated using

$$
\delta_{1}=2[\mu \mathrm{F}(\mu)-\psi(\mu)] \text { and } \delta_{2}=\mu-2 \psi(M),
$$

where

$$
\psi(q)=(1+\lambda) \theta \gamma\left[-\frac{1}{\alpha}+1,\left(\frac{\theta}{q}\right)^{\alpha}\right]-\lambda \gamma\left[-\frac{1}{\alpha}+1,2\left(\frac{\theta}{q}\right)^{\alpha}\right] .
$$

The function $\psi(q)$ is used to determine the Bonferroni and Lorenz curves [5], which have applications in econometrics and finance, reliability and survival analysis, demography, insurance and biomedical sciences. By using (25), one obtains the Bonferroni curve

$$
\mathrm{B}(\mathrm{P})=\frac{1}{\mathrm{P \mu}}\left\{(1+\lambda) \theta \gamma\left[-\frac{1}{\alpha}+1,\left(\frac{\theta}{\mathrm{q}}\right)^{\alpha}\right]-\lambda \gamma\left[-\frac{1}{\alpha}+1,2\left(\frac{\theta}{\mathrm{q}}\right)^{\alpha}\right]\right\},
$$

and the Lorenz curve

$$
L(P)=\frac{1}{\mu}\left\{(1+\lambda) \theta \gamma\left[-\frac{1}{\alpha}+1,\left(\frac{\theta}{q}\right)^{\alpha}\right]-\lambda \gamma\left[-\frac{1}{\alpha}+1,2\left(\frac{\theta}{q}\right)^{\alpha}\right]\right\} .
$$

\section{Order statistics}

The density of the rth order statistic $X_{(r)}$ of a random sample drawn from the $\operatorname{TIW}(x ; \alpha, \theta, \lambda)$ distribution with $|\lambda| \leqslant 1$, follows from Arnold et al. [1], and is

$$
f_{r: n}(x)=\frac{F(x)^{r-1}[1-F(x)]^{n-r} f(x)}{B(r, n-r+1)}, \quad x>0 .
$$


By setting $\Psi=\exp \left[-\left(\frac{\theta}{\chi}\right)^{\alpha}\right]$, substituting (4) and (6) into (26), we obtain

$$
\begin{aligned}
f_{r: n}(x)= & n C_{n-1: r-1} \sum_{i=0}^{n-r} C_{n-r: i}(-1)^{i}[\Psi(1+\lambda-\lambda \Psi)]^{r+i-1} \alpha \theta^{\alpha-\frac{1}{\alpha}-1} \\
& \times\left[\log \left(\frac{1}{\Psi}\right)\right]^{\frac{1}{\alpha}+1} \varepsilon
\end{aligned}
$$

where $\varepsilon=\Psi(1+\lambda-2 \lambda \Psi)$.

Combining terms of the order statistics of the TIW distribution,

$$
f_{r: n}(x)=n C_{n-1: r-1} \sum_{i=0}^{n-r} \sum_{j=0}^{\infty} \xi_{i, j, r, \lambda} \curlyvee(\alpha, \theta, \Psi, r, i, j, \lambda),
$$

where

$$
\xi_{i, j, r, \lambda}=n C_{n-r: i} C_{r+i-1: j}(-1)^{i+j}(1+\lambda)^{r+i-1}\left(\frac{\lambda}{1+\lambda}\right)^{j}
$$

and

$$
\curlyvee(\alpha, \theta, \Psi, r, i, j, \lambda)=\alpha \theta^{\alpha-\frac{1}{\alpha}-1}\left[\log \left(\frac{1}{\Psi}\right)\right]^{\frac{1}{\alpha}+1} \Psi^{r+i+j}(1+\lambda-2 \lambda \Psi)
$$

By (27), the kth moment of the rth order statistics $X_{(r)}$ is

$$
\mu_{\mathrm{k}}^{\mathrm{n}-\mathrm{r}}=\mathrm{nC}_{\mathrm{n}-1: \mathrm{r}-1} \sum_{i=0}^{\mathrm{n}-\mathrm{r}} \sum_{j=0}^{\infty} \xi_{i, j, r, \lambda} \theta^{\mathrm{k}} \Gamma\left(1-\frac{\mathrm{k}}{\alpha}\right) \mathfrak{I}(\alpha, \lambda, r, i, j, k),
$$

where $\mathfrak{I}(\alpha, \lambda, r, i, j, k)=(1+\lambda)(r+i+j)^{\frac{k}{\alpha}-1}-2 \lambda(r+i+j+1)^{\frac{k}{\alpha}-1}$.

\section{$7 \quad$ Bladder cancer application}

In this section, we test the performance of the TIW distribution and show it to be an improved model as compared to some of its sub-models such 
Table 1: Summary statistics for bladder cancer remission times

\begin{tabular}{cccccc} 
Application & Mean & Median & SD & Skewness & kurtosis \\
\hline Data & 9.37 & 6.40 & 10.51 & 3.33 & 16.15 \\
Model & QD & Median & CQD & Bowley & Moors \\
TIW & 6.72 & 5.45 & 0.74 & 0.54 & 2.46 \\
IW & 7.49 & 5.31 & 0.78 & 0.57 & 2.71
\end{tabular}

Table 2: Estimated parameters of the TIW distribution for remission times of bladder cancer patients

Parameter ML Estimates Standard Error t-stat p-value

\begin{tabular}{ccccc}
\hline$\alpha$ & 0.84 & 0.05 & 17.80 & $4.39 \times 10^{-36}$ \\
$\theta$ & 1.71 & 0.22 & 7.62 & $5.42 \times 10^{-12}$ \\
$\lambda$ & -0.86 & 0.093 & -9.17 & $1.20 \times 10^{-15}$
\end{tabular}

as the TIR, TIE and IW distributions. We consider the survival times of 128 bladder cancer patients to see how the new model works in practice. The data was obtained from Lee and Wang [11, pg. 231]. We fitted the TIW, TIR, TIE and IW distributions by the method of maximum likelihood. The required numerical evaluations are implemented in $\mathrm{R}$ [14]. The summary statistics of the bladder cancer data and the median, coefficient of quartile deviation (CQD), Bowley skewness and Moors kurtosis for the TIW and IW distributions are given in Table 1. Using the maximum likelihood estimates the approximate $95 \%$ two sided confidence interval for the parameters $\alpha$, $\theta$ and $\lambda$ are $[0.74,0.93],[1.27,2.15]$ and $[-1.04,-0.67]$, respectively.

The parameter estimates with their standard errors and associated $t$-statistics and $p$-values are given in Table 2. The parameter estimates with the Akaike information criteria (AIC), BIC (Bayesian information criteria), MSE (mean square error) and the associated Kolmogorov-Smirnov (K-S) values for these distributions are given in Table 3. The TIW distribution has the lowest AIC, BIC, MSE and K-S values, indicating that the proposed distribution provides a better fit than the other three lifetime distributions. 
Table 3: Estimates of the model parameters for bladder cancer data and the AIC, BIC, MSE and associated K-S values

\begin{tabular}{ccccc} 
Distribution & TIW & TIR & TIE & IW \\
\hline$\alpha$ & 0.846 & - & - & 0.752 \\
$\theta$ & 1.71 & 0.75 & 1.68 & 3.26 \\
$\lambda$ & -0.856 & -0.954 & -0.860 & - \\
AIC & 879.4 & 1424.4 & 889.6 & 892.0 \\
BIC & 879.7 & 1424.6 & 889.8 & 892.2 \\
MSE & 0.074 & 0.445 & 0.092 & 0.088 \\
K-S & 0.119 & 0.676 & 0.155 & 0.131
\end{tabular}

Table 4: The ML estimation, the values of the log-likelihood function, degrees of freedom (d.f) and p-values for bladder cancer remission times.

\begin{tabular}{cccccc} 
Distribution & $\mathrm{H}_{0}$ & $\log \mathrm{L}(\cdot)$ & $\lambda$ & d.f. & p-value \\
\hline TIR & $\alpha=2$ & -710.2 & 547.04 & 1 & 0.0000 \\
TIE & $\alpha=1$ & -442.8 & 12.20 & 1 & 0.0005 \\
IW & $\lambda=0$ & -444.0 & 14.65 & 1 & 0.0001
\end{tabular}

We perform the following likelihood ratio tests $\mathrm{H}_{0}$ to test the hypotheses

- $\alpha=2$, or $x_{1}, x_{2}, \ldots, x_{n} \sim$ TIR.

- $\alpha=1$, or $x_{1}, x_{2}, \ldots, x_{n} \sim$ TIE.

- $\lambda=0$, or $x_{1}, x_{2}, \ldots, x_{n} \sim$ IW.

From Table 4 we reject the null hypothesis in all cases in favour of the TIW distribution. Figure 5 illustrates the fitted TIW and IW densities and survival functions along with the conventional Kaplan-Meier curve [10]. The fitted survival plot shows that the TIW distribution allows a closer approach to the empirical survival function. This suggests that the TIW distribution has greater flexibility and allows a better fit to bladder cancer data. The AIC and BIC suggest that the improved fit is beneficial and takes account of the 
greater complexity of the TIW distribution.

\section{Conclusion}

We proposed a new lifetime model named the TIW distribution that is shown to perform better than the TIR, TIE and IW distributions. The characterisations of the TIW distribution are studied. The instantaneous failure patterns show that the TIW distribution has increasing and decreasing failure rates for life time data. The utility of the TIW distribution is illustrated using ML estimation. We provide a mathematical treatment of the TIW distribution including the density and the kth moment of the rth order statistics. We consider likelihood ratio tests to compare the TIW distribution with its submodels to show the model's utility. The AIC, BIC, MSE and K-S tests show that the TIW distribution provides better fit for life time data.

Acknowledgements The authors are grateful to the referee for helpful comments and suggestions.

\section{References}

[1] Arnold, B. C., Balakrishnan A. N. and Nagaraja H. N., A first course in order statistics, Wiley, New York, 1992. doi:10.1002/9781118150412 $\mathrm{C} 210$

[2] Aryal, G. R. and Tsokos, C. P., Transmuted Weibull distribution: A generalization of the Weibull Probability distribution. Europe. J. of Pure Appl. Math., 4(2):89-102, 2011.

http://www.ejpam.com/index.php/ejpam/article/view/1170 C199 


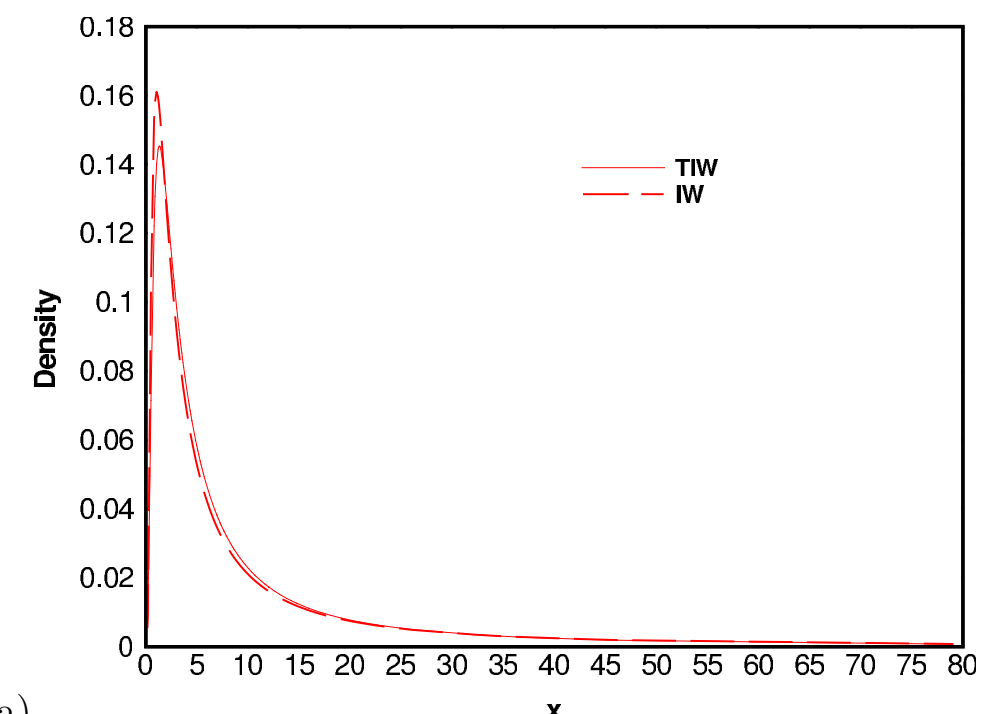

(a)

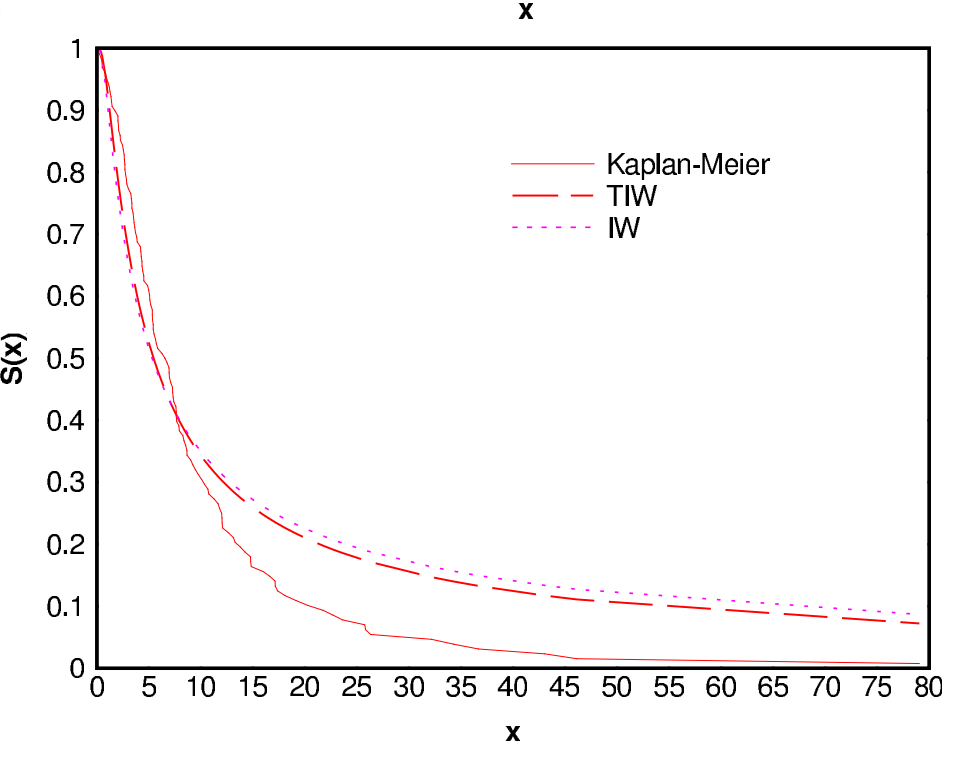

Figure 5: Fitted (a) density; and (b) survival functions for bladder cancer remission times (in months) of the TIW and IW distributions. 
[3] Calabria, R. and Pulcini, G., On the maximum likelihood and least-squares estimation in the inverse Weibull distribution. Stat. Appl., 2:53-66, 1990. http://sa-ijas.stat.unipd.it/sites/sa-ijas. stat.unipd.it/files/53-66.pdf C198

[4] de Gusmão, F. R. S., Ortega, E. M. M. and Cordeiro, G. M., The generalized inverse Weibull distribution. Stat. Pap., 52:591-619, 2011. doi:10.1007/s00362-009-0271-3 C199

[5] Cordeiro, G. M., Gomes, A. E., da-Silva, C. Q. and Ortega, E. M. M., The beta exponentiated Weibull distribution. J. Stat. Comput. Sim., 83(1):114-138, 2013. doi:10.1080/00949655.2011.615838 C210

[6] Havrda, J. and Charvát, F., Quantification method in classification processes: concept of structural $\alpha$-entropy. Kybernetika, 3:30-35, 1967. http://www. kybernetika.cz/content/1967/1/30 C209

[7] Khan, M. S. and King, R., Transmuted modified Weibull distribution: A generalization of the modified Weibull probability distribution. Europe. J. of Pure Appl. Math., 6(1):66-88, 2013. http://www.ejpam.com/index.php/ejpam/article/view/1606 C199

[8] Khan, M. S. and King, R., Transmuted generalized inverse Weibull distribution. J. Appl. Stat. Sci., 20(3):15-32, 2013. https://www .novapublishers.com/catalog/product_info.php? products_id=47370 C199

[9] Keller, A. Z., Kamath A. R. R. and Perera, U. D., Reliability analysis of CNC machine tools. Reliab. Eng., 3:449-473, 1982. doi:10.1016/0143-8174(82)90036-1 C198

[10] Kaplan, E. L. and Meier, P., Nonparametric estimation from incomplete observations. J. Am. Stat. Assoc., 53(282):457-481, 1958. doi:10.1080/01621459.1958.10501452 C213

[11] Lee, E. T. and Wang, J. W., Statistical Methods for Survival Data Analysis. Wiley, New York, 2003. doi:10.1002/0471458546 C212 
[12] Liu, C.-C., A Comparison between the Weibull and Lognormal Models used to Analyze Reliability Data. PhD Thesis University of Nottingham, 1997. C204

[13] Rényi, A., On measures of information and entropy. Proc. Fourth Berkeley Symp. on Math. Statist. and Prob. 1:547-561, 1961. http://projecteuclid.org/euclid.bsmsp/1200512181 C209

[14] The R Project for Statistical Computing, Vienna, Austria, 2014. http://www.R-project.org C212

[15] Shaw, W. T. and Buckley, I. R. C., The alchemy of probability distributions: beyond Gram-Charlier expansions, and a skew-kurtotic normal distribution from a rank transmutation map. Technical report, 2009. http://arxiv.org/abs/0901.0434 C199

\section{Author addresses}

1. M. Shuaib Khan, School of Mathematical and Physical Sciences, University of Newcastle, Callaghan, NSW 2308, Australia. mailto:muhammad.s.khan@newcastle.edu.au

2. Robert King, School of Mathematical and Physical Sciences, University of Newcastle, Callaghan, NSW 2308, Australia.

3. Irene L. Hudson, School of Mathematical and Physical Sciences, University of Newcastle, Callaghan, NSW 2308, Australia. 